\title{
AN EXTENSION OF NORM INEQUALITIES FOR INTEGRAL OPERATORS ON CONES WHEN $0<p<1$
}

\author{
M. V. SIADAT AND K. ZHOU
}

(Communicated by Palle E. T. Jorgensen)

\begin{abstract}
We extend our recent results concerning norm inequalities on cones to include the case when $0<p<1$.
\end{abstract}

In this note, we let $V$ be a homogeneous cone in $R^{n} \cdot V$ defines a partial ordering in $R^{n}$ in such a way that $x<_{V} y$ if and only if $y-x \in V$. The cone interval $\langle a, b\rangle$ is thus given by $\langle a, b\rangle=\left\{x \in V: a<_{V} x<_{V} b\right\}$. For $x \in V$ we define $\Delta_{V}(x)=\int_{\langle 0, x\rangle} d y$.

Let $G(V)$ denote the automorphism group of $V$ and $f: V \rightarrow R^{+}$be a $V$-homogeneous function of order $\beta$. It is known (see $[2,5])$ that if $f(x)$ is not identically 0 then $f(x)=c\left(\Delta_{V}(x)\right)^{\beta}$ for all $x \in V$.

A *-function on $V$ is a mapping $x \rightarrow x^{*}$ such that $x^{*}=-\operatorname{grad} \log \phi_{V}(x)$, where $\phi_{V}(x)$ is the characteristic function of $V$. We have (see [1,4]) that $\left(x^{*}\right)^{*}=x$ and the Jacobian determinant $\left|\partial_{x} x^{*}\right|=c \Delta_{V}^{-2}(x)$, where $c$ is a constant depending on $V$.

Let $V^{*}$ be the dual of $V$ and $G\left(V \rightarrow V^{*}\right)$ be the group of linear transforms mapping $V$ onto $V^{*}$. A homogeneous cone $V$ is called a domain of positivity if there is an $S \in G\left(V \rightarrow V^{*}\right)$ so that $S$ is symmetric and positive definite. It can be shown (see $[4,5])$ that if $V$ is a domain of positivity then $x<_{V} y \Leftrightarrow$ $y^{*}<V^{*} x^{*}$.

We shall continue to consider integral operators of the form

$$
K f(x)=\int_{V} k(x, y) f(y) d y, \quad x \in V,
$$

and

$$
K^{*} f(y)=\int_{V} k(x, y) f(x) d x, \quad y \in V,
$$

where $f: V \rightarrow R^{+}$and $k: V \times V \rightarrow R^{+}$is $(V \times V)$-homogeneous of order $\beta$; that is,

$$
k(A x, A y)=|A|^{\beta} k(x, y) \quad \forall A \in G(V) .
$$

Received by the editors February 26, 1992; presented at the Regional Meeting of the AMS in Portland, Oregon, June 1991.

1991 Mathematics Subject Classification. Primary 44A15.

Key words and phrases. Homogeneous cone, domain of positivity, Hardy's inequality. 
We have shown (see [5]) the following general theorem concerning the $L^{p}$ boundedness of an integral operator on a cone:

Theorem 1. Let $V$ be a homogeneous cone in $R^{n}$ and $1 \leq p \leq q<\infty$. Assume that the kernel $k(x, y): V \times V \rightarrow R^{+}$is $(V \times V)$-homogeneous of order $\beta$. If, for some $\alpha, \gamma \in R, K \Delta_{V}^{\alpha}(x)<\infty$ and

$$
\int_{V} k^{q / p}(x, y) \Delta_{V}^{\gamma-q+(\alpha+\beta+1) q / p^{\prime}}(x) d x<\infty
$$

for $x, y \in V$, where $1 / p+1 / p^{\prime}=1$, then

$$
\left(\int_{V} \Delta_{V}^{\gamma-q}(x)(K f(x))^{q} d x\right)^{1 / q} \leq c\left(\int_{V} f^{p}(x) \Delta_{V}^{\beta p+(\gamma+1) p / q-1}(x) d x\right)^{1 / p} .
$$

In this note, we extend the preceding result to the case $0<p<1$.

Theorem 2. Let $V \subset R^{n}$ be a homogeneous cone and $0<p<1$. Assume that $k(x, y): V \times V \rightarrow R^{+}$is $(V \times V)$-homogeneous of order -1 . If there exist $\alpha, \gamma \in R$ such that

$$
K \Delta_{V}^{\alpha}(x)<\infty
$$

and

$$
K^{*} \Delta_{V}^{\gamma-p+\left(\alpha p / p^{\prime}\right)}(y)<\infty
$$

for $x, y \in V$, then

$$
\int_{V} \Delta_{V}^{\gamma-p}(x)(K f(x))^{p} d x \geq c \int_{V} f^{p}(x) \Delta_{V}^{\gamma-p}(x) d x,
$$

in the sense that if the integral on the left is finite, then the integral on the right is also finite and the inequality holds.

Proof. We show first that, for some constant $c>0$,

$$
(K f(x))^{p} \geq c \cdot K\left(f^{p} \cdot \Delta_{V}^{-\alpha p / p^{\prime}}\right)(x) \cdot \Delta_{V}^{\alpha p / p^{\prime}}(x) .
$$

In fact, since $(1 / p)>1$, we use Hölder's inequality to obtain that

$$
\begin{aligned}
\int_{V} k(x, y) f^{p}(y) \Delta_{V}^{-\alpha p / p^{\prime}}(y) d y \\
\quad=\int_{V}(k(x, y) f(y))^{p} \cdot k^{-p / p^{\prime}}(x, y) \Delta_{V}^{-\alpha p / p^{\prime}}(y) d y \\
\quad \leq\left(\int_{V} k(x, y) f(y) d y\right)^{p} \cdot\left(\int_{V}\left(k^{-p / p^{\prime}}(x, y) \Delta_{V}^{-\alpha p / p^{\prime}}(y)\right)^{1 /(1-p)} d y\right)^{1-p} \\
\quad=(K f(x))^{p} \cdot\left(K \Delta_{V}^{\alpha}(x)\right)^{1-p} .
\end{aligned}
$$

Note that, because of assumption (1), $K \Delta_{V}^{\alpha}(x)$ is $V$-homogenous of order $\alpha$, and so we have $K \Delta_{V}^{\alpha}(x)=c \cdot \Delta_{V}^{\alpha}(x)$, for some constant $c$. Therefore,

$$
\int_{V} k(x, y) f^{p}(y) \Delta_{V}^{-\alpha p / p^{\prime}}(y) d y \leq c \cdot(K f(x))^{p} \cdot \Delta_{V}^{\alpha(1-p)}(x),
$$

and then we have (4). 
Using (4), we have that

$$
\begin{aligned}
\int_{V} \Delta_{V}^{\gamma-p}(x)(K f(x))^{p} d x \\
\quad \geq c \cdot \int_{V} \Delta_{V}^{\gamma-p}(x)\left(\Delta_{V}^{\alpha}(x)\right)^{p / p^{\prime}}\left(\int_{V} k(x, y) f^{p}(y) \Delta_{V}^{-\alpha p / p^{\prime}}(y) d y\right) d x \\
\quad=c \cdot \int_{V} f^{p}(y) \Delta_{V}^{-\alpha p / p^{\prime}}(y)\left(\int_{V} \Delta_{V}^{\gamma-p+\left(\alpha p / p^{\prime}\right)}(x) k(x, y) d x\right) d y
\end{aligned}
$$

Note that, because of $(2), K^{*} \Delta_{V}^{\gamma-p+\left(\alpha p / p^{\prime}\right)}(y)$ is $V$-homogeneous of degree $\gamma-p+\left(\alpha p / p^{\prime}\right)$, and hence we have $K^{*} \Delta_{V}^{\gamma-p+\left(\alpha p / p^{\prime}\right)}(y)=c \Delta_{V}^{\gamma-p+\left(\alpha p / p^{\prime}\right)}(y)$, for some constant $c$. Therefore,

$$
\begin{gathered}
\int_{V} f^{p}(y) \Delta_{V}^{-\alpha p / p^{\prime}}(y)\left(\int_{V} \Delta_{V}^{\gamma-p+\left(\alpha p / p^{\prime}\right)}(x) k(x, y) d x\right) d y \\
=c \int_{V} f^{p}(y) \Delta_{V}^{\gamma-p}(y) d y
\end{gathered}
$$

and thus (3) holds.

Let $\Sigma=\{x \in V:|x|=1\}$. Define $\sigma_{0}(V)=\inf \left\{\alpha \in R: \int_{\Sigma} \Delta_{V}^{\alpha}(t) d t<\infty\right\}$ and $\sigma(V)=\max \left\{-1, \sigma_{0}\right\}$. It is known (see [2]) that if $\alpha>\sigma(V)$, then $\int_{\langle 0, x\rangle} \Delta_{V}^{\alpha}(y) d y<\infty$ for $x \in V$.

We have the following generalization of Hardy's inequality in the case $0<$ $p<1$.

Theorem 3. Let $V$ be a domain of positivity in $R^{n}$ and $0<p<1$. If $\gamma>$ $\left(1+\sigma\left(V^{*}\right)\right)(p-1)+\sigma(V)+p$, then

$$
\int_{V} \Delta_{V}^{\gamma-p}(x)\left(\int_{\langle x, \infty\rangle} f(y) \Delta_{V}^{-1}(y) d y\right)^{p} d x \geq c \int_{V} f^{p}(x) \Delta_{V}^{\gamma-p}(x) d x .
$$

Proof. Let $k(x, y)=\Delta_{V}^{-1}(y) \chi_{\langle x, \infty\rangle}(y)$ for $x, y \in V$. Clearly, $k(x, y)$ is $(V \times$ $V)$-homogeneous of order -1 . Let $\gamma$ be given so that $\gamma>\left(1+\sigma\left(V^{*}\right)\right) \times$ $(p-1)+\sigma(V)+p$. It follows that $\left(p^{\prime} / p\right)(\gamma-\sigma(V)-p)<1+\sigma\left(V^{*}\right)$. Let $\alpha$ be a number so that $\alpha<-1-\sigma\left(V^{*}\right)$.

Since $V$ is a domain of positivity, $x<_{V} y \Leftrightarrow y^{*}<_{V^{*}} x^{*}$. Note that $\Delta_{V}(y)=$ $c \cdot \Delta_{V^{*}}^{-1}\left(y^{*}\right)$ and $\partial_{y^{*}} y=c \cdot \Delta_{V^{*}}^{-2}\left(y^{*}\right)$. So if we introduce a change of variable $z=y^{*}$, then we have that

$$
\begin{aligned}
K \Delta_{V}^{\alpha}(x) & =\int_{V} \Delta_{V}^{-1}(y) \chi_{\langle x, \infty\rangle}(y) \Delta_{V}^{\alpha}(y) d y \\
& =\int_{\langle x, \infty\rangle} \Delta_{V}^{-1+\alpha}(y) d y=c \int_{\left\langle 0, x^{*}\right\rangle} \Delta_{V^{*}}^{-1-\alpha}(z) d z
\end{aligned}
$$

By the choice of $\alpha$ the last integral is finite for any $x \in V$. 
We also have that

$$
\begin{aligned}
K^{*} \Delta_{V}^{\gamma-p+\left(\alpha p / p^{\prime}\right)}(y) & =\int_{V} \Delta_{V}^{-1}(y) \Delta_{V}^{\gamma-p+\left(\alpha p / p^{\prime}\right)}(x) \chi_{\langle x, \infty)}(y) d x \\
& =\Delta_{V}^{-1}(y) \int_{\langle 0, y\rangle} \Delta_{V}^{\gamma-p+\left(\alpha p / p^{\prime}\right)}(x) d x .
\end{aligned}
$$

Note that since $-\left(p^{\prime} / p\right)(\gamma-\sigma(V)-p)>\alpha$ and $p^{\prime}<0$, we have $\gamma>\sigma(V)+$ $p-\left(\alpha p / p^{\prime}\right)$. Thus the last integral above is finite for any $y \in V$.

By Theorem 2, we conclude that

$$
\int_{V} \Delta_{V}^{\gamma-p}(x)\left(\int_{\langle x, \infty\rangle} f(y) \Delta_{V}^{-1}(y) d y\right)^{p} d x \geq c \int_{V} f^{p}(x) \Delta_{V}^{\gamma-p}(x) d x .
$$

Theorem 4. Let $V$ be a domain of positivity in $R^{n}$ and $0<p<1$. If $\gamma<$ $(1-\sigma(V))(p-1)-\sigma\left(V^{*}\right)$, then

$$
\int_{V} \Delta_{V}^{\gamma-p}(x)\left(\frac{1}{\Delta_{V}(x)} \int_{\langle 0, x\rangle} f(y) d y\right)^{p} d x \geq c \int_{V} f^{p}(x) \Delta_{V}^{\gamma-p}(x) d x .
$$

Proof. Let $k(x, y)=\Delta_{V}^{-1}(x) \chi_{\langle 0, x\rangle}(y)$ for $x, y \in V$. Clearly, $k(x, y)$ is $(V \times$ $V)$-homogeneous of order -1 . Let $\gamma$ be given so that $\gamma<(1-\sigma(V)) \times$ $(p-1)-\sigma\left(V^{*}\right)$. It follows that $\left(p^{\prime} / p\right)\left(-\gamma+p-\sigma\left(V^{*}\right)-1\right)<\sigma(V)$. Let $\alpha$ be a number so that $\alpha>\sigma(V)$. Then it follows that $-\gamma+p-\left(\alpha p / p^{\prime}\right)-1>\sigma\left(V^{*}\right)$.

Since $V$ is a domain of positivity, $x<_{V} y \Leftrightarrow y^{*}<V^{*} x^{*}$. Note also that $\Delta_{V}(x)=c \cdot \Delta_{V^{*}}^{-1}\left(x^{*}\right)$ and $\partial_{x^{*}} x=c \cdot \Delta_{V^{*}}^{-2}\left(x^{*}\right)$. So if we introduce a change of variable $z=x^{*}$, we then have that

$$
\begin{aligned}
K^{*} \Delta_{V}^{\gamma-p+\left(\alpha p / p^{\prime}\right)}(y) & =\int_{V} \Delta_{V}^{-1}(x) \chi\langle 0, x\rangle(y) \Delta_{V}^{\gamma-p+\left(\alpha p / p^{\prime}\right)}(x) d x \\
& =\int_{\langle y, \infty\rangle} \Delta_{V}^{\gamma-p+\left(\alpha p / p^{\prime}\right)-1}(x) d x=c \int_{\left\langle 0, y^{*}\right\rangle} \Delta_{V^{*}}^{-\gamma+p-\left(\alpha p / p^{\prime}\right)-1}(z) d z .
\end{aligned}
$$

Because $-\gamma+p-\left(\alpha p / p^{\prime}\right)-1>\sigma\left(V^{*}\right)$, the last integral is finite for any $y \in V$.

We also have that

$$
K \Delta_{V}^{\alpha}(x)=\Delta_{V}^{-1}(x) \int_{\langle 0, x\rangle} \Delta_{V}^{\alpha}(y) d y .
$$

Because $\alpha>\sigma(V)$, the integral above is finite for any $x \in V$.

By Theorem 2, we conclude that

$$
\int_{V} \Delta_{V}^{\gamma-p}(x)\left(\frac{1}{\Delta_{V}(x)} \int_{\langle 0, x\rangle} f(y) d y\right)^{p} d x \geq c \int_{V} f^{p}(x) \Delta_{V}^{\gamma-p}(x) d x
$$

\section{REFERENCES}

1. M. Koecher, Positivitätsbereiche im $R^{n}$, Amer. J. Math. 79 (1957), 575-596. (German)

2. T. Ostrogorski, Analogues of Hardy's inequality in $R^{n}$, Studia Math. 88 (1988), 209-219.

3. R. T. Rockafellar, Convex Analysis, Princeton Univ. Press, Princeton, NJ, 1970. 
4. O. Rothaus, Domains of positivity, Abh. Math. Sem. Univ. Hamburg 24 (1960), 189-235.

5. Y. Sagher, M. V. Siadat, and K. Zhou, Norm inequalities for integral operators on cones, Colloq. Math. 60/61 (1990).

Department of Mathematics, California State University, Domingues Hills, Carson, CALIFORNIA 90747

Department of Mathematics, California State University, Sacramento, California 95819 